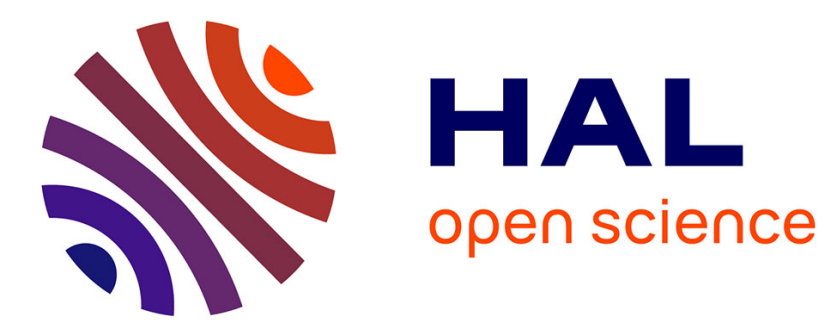

\title{
Morphology of Calcium Oxalate Polyhydrates: A Quantum Chemical and Computational Study
}

Theau Debroise, Thomas Sedzik, Jelle Vekeman, Yangyang Su, Christian Bonhomme, Frederik Tielens

\section{- To cite this version:}

Theau Debroise, Thomas Sedzik, Jelle Vekeman, Yangyang Su, Christian Bonhomme, et al.. Morphology of Calcium Oxalate Polyhydrates: A Quantum Chemical and Computational Study. Crystal Growth \& Design, 2020, 20 (6), pp.3807-3815. 10.1021/acs.cgd.0c00119 . hal-02890692

\section{HAL Id: hal-02890692 https://hal.science/hal-02890692}

Submitted on 28 Aug 2020

HAL is a multi-disciplinary open access archive for the deposit and dissemination of scientific research documents, whether they are published or not. The documents may come from teaching and research institutions in France or abroad, or from public or private research centers.
L'archive ouverte pluridisciplinaire HAL, est destinée au dépôt et à la diffusion de documents scientifiques de niveau recherche, publiés ou non, émanant des établissements d'enseignement et de recherche français ou étrangers, des laboratoires publics ou privés. 


\section{Morphologies of Calcium Oxalate Polyhydrates:}

A Quantum Chemical and Computational Study

Théau Debroise ${ }^{1}$, Thomas Sedzik ${ }^{1}$, Jelle Vekeman ${ }^{2}$, Yangyang Su ${ }^{2}$, Christian Bonhomme ${ }^{1}$ and Frederik Tielens ${ }^{2, *}$

${ }^{1}$ Sorbonne Université, CNRS, Laboratoire Chimie de la Matière Condensée de Paris, LCMCP, 4 Place Jussieu, F-75005 Paris, France

${ }^{2}$ General Chemistry (ALGC), Materials Modeling Group, Vrije Universiteit Brussel (Free University Brussels-VUB), Pleinlaan 2, 1050 Brussel, Belgium

*Author to whom correspondence should be addressed: frederik.tielens@,vub.be 


\section{$\underline{\text { Abstract }}$}

Monohydrated and dihydrated calcium oxalate have been widely studied in the literature because of their role in urolithiasis, a mammal pathology responsible for the formation of stones in the kidney. It is clear that the physicochemical environment plays a crucial role in the crystal growth and the resulting morphologies of calcium oxalates. To study these processes, reliable models for the calcium oxalate's faces, exposed to water and potential additives, are needed. Here, we have used a total surface energy minimization approach to predict the crystal morphology of the calcium oxalate monohydrate and dihydrate phases. Surface energies were calculated at density functional theory level, taking into account surface relaxation and the effect of solvation. An excellent agreement was found between theoretically predicted morphologies and their experimental counterparts obtained by SEM, clearly demonstrating the importance of the inclusion of water in the model for the prediction of morphologies. 


\section{Introduction}

Calcium oxalate polyhydrates are ionic crystals present in minerals and living organisms. They are, for example, found in most plant families under the name raphides, ${ }^{1}$ where they are associated with diverse functions such as protection and structure. ${ }^{2}$ Aside from their role in plant biology, calcium oxalates have also been extensively studied because of their role in urolithiasis. This pathology originates from the formation of stones in the urinary system, called kidney stones. ${ }^{3,4}$ The main constituents of kidney stones are now established and correspond to a limited number of well-defined crystalline phases: calcium oxalates (with different hydration rates), ammonium, calcium and magnesium phosphates and uric acid. In recent decades, calcium oxalate-based stones are the most frequently observed in Europe and North America (> $70 \%$ of all samples analyzed). ${ }^{3,4}$

From a crystallographic point of view, the calcium oxalate family is classified according to its hydration rate: anhydrous calcium oxalate (ACO), whewellite $\left(\mathrm{CaC}_{2} \mathrm{O}_{4} \cdot \mathrm{H}_{2} \mathrm{O} ; \mathrm{COM}\right)$, weddellite $\left(\mathrm{CaC}_{2} \mathrm{O}_{4} \cdot 2 \mathrm{H}_{2} \mathrm{O}\right.$; COD) and caoxite $\left(\mathrm{CaC}_{2} \mathrm{O}_{4} \cdot 3 \mathrm{H}_{2} \mathrm{O}\right.$; COT), while an amorphous phase has also been described in nanoparticles. ${ }^{5}$ In this study we focus on COM and COD as they are the two phases observed in kidney stones. ${ }^{4,6}$ Previously, the COM structure has been resolved by X-Ray and neutron diffraction,7-12 suggesting two polymorphs: a first structure stable in the range $318 \mathrm{~K}-425 \mathrm{~K}$ and a second in the range $293 \mathrm{~K}-318 \mathrm{~K}$. As kidney stones are formed at physiological temperature $(\approx 310 \mathrm{~K})$, only the second structure was considered in this work. It is monoclinic and belongs to the space group $\mathrm{P} 2{ }_{1} / c$. The COD structure was also previously resolved by X-ray diffraction and is a tetragonal structure belonging to the space group $\mathrm{I} 4 / \mathrm{m}^{9,13,14}$

COM has been observed by Scanning Electron Microscopy (SEM) in several in vitro syntheses in aqueous medium. ${ }^{15-18}$ Four faces came out as predominant from these studies: (010), (100), $(021)$ and $(12 \overline{1}) \cdot{ }^{9,16}$ The $(010)$ and (100) faces are always observed, while either the $(021)$ or $(12 \overline{1})$ face is always present as well, sometimes both of them. The corresponding morphologies are presented in Table 1, together with the conditions leading to their appearance and corresponding references. As mentioned before, COM morphologies have been observed in kidney stones where they present morphologies similar to those presented in Table 1a and

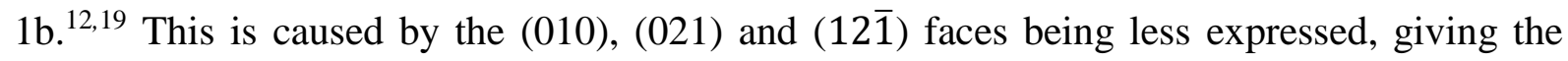


crystals a flat aspect, while they are stacked on the (100) face with proteins between them. ${ }^{19}$ Compared to the synthetic and kidney stone whewellite, mineral whewellite crystals exhibit a

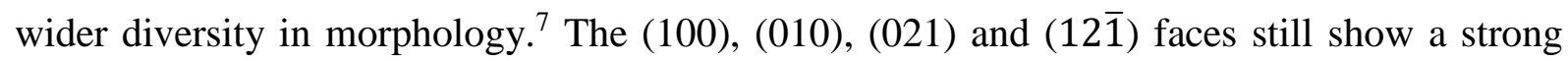
occurrence, but the (001) face becomes the most exposed face in minerals. A large variety of other faces is observed more rarely in few crystals: (140), (13 $\overline{1}),(12 \overline{3}),(14 \overline{1}),(161),(140)$,

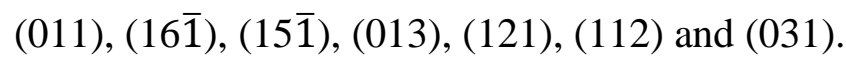

Table 1: COM morphologies observed by SEM for different synthetic procedures ${ }^{16-18,20}$

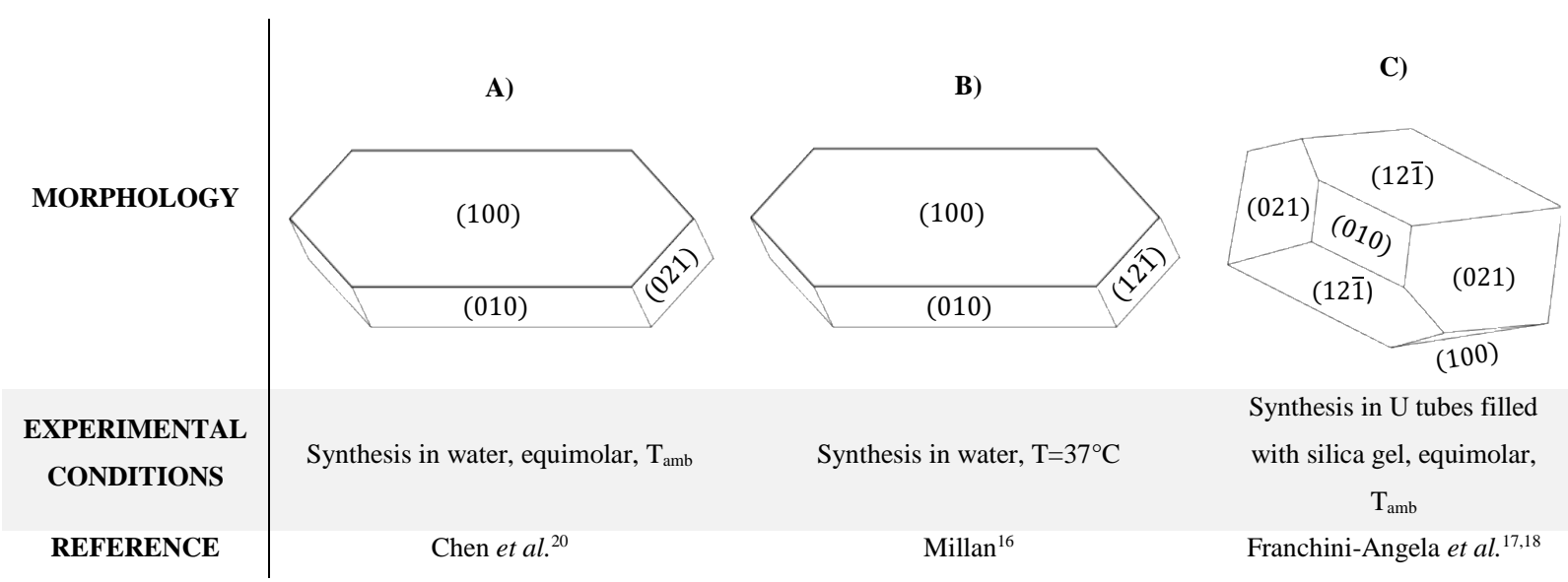

The Hartman-Perdok (HP) theory ${ }^{21}$, the Bravais-Friedel-Donnay-Harker (BFDH) law ${ }^{22}$, the Ising Model $^{23}$ and the attachment energy method ${ }^{24}$ have been used by Millan ${ }^{16}$ to predict COM morphology. According to the HP theory, only F faces (faces with a PBC in two dimensions) are exposed as they grow slower because they need to form strong bonds in two dimensions.

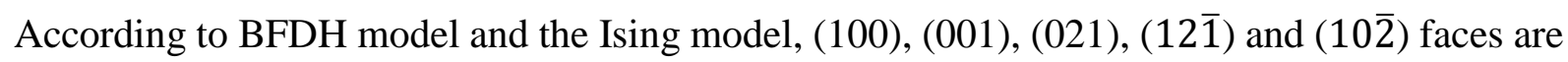
predicted in COM, while the attachment theory predicts the same faces, except for the $(10 \overline{2})$ face. Tommasso et al..$^{25}$ have predicted COM morphology in a force field study and found a stabilization of the (100) face due to a solvation effect (pure water). Such a stabilization is not observed on the (001) face, possibly explaining why the (001) face is not observed in aqueous conditions experimentally.

Except for the last study, the main difference between theory and experiment originates from the (001) face which is always predicted theoretically, but never observed in syntheses nor in kidney stones. This may be due to the growth kinetics. Indeed, Milan ${ }^{16}$ observed that faces

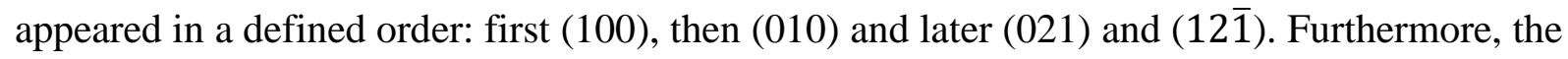
(001) face is prevalent in minerals ${ }^{7,16}$ suggesting that this face may take more time to appear. 
COD morphologies have also been reported in many in vitro studies and are shown in Table 2, together with the experimental conditions in which they occur and the appropriate references. As shown in Table 2a and 2c, COD crystals often have the form of a bipyramid dominated by (101) faces. Under certain conditions, the (010) face is intercalated between the two pyramids formed by the (101) faces as shown in Table 2b. ${ }^{20,26,27,28}$ Although COD is less observed in kidney stones than COM, it is found in a bipyramid morphology with the (101) face dominating as are the COD crystals found in marine sediments. ${ }^{29,30,31}$. As COM, COD morphologies have been predicted in the literature by HP theory, the BFDH law and the attachment energy method..$^{31} \mathrm{HP}$ analysis predicts the existence of five F-type faces: (110), (101), (010), (121) and (211), while from attachment energy analysis, only three faces are expressed: (101), (010) and (110). The difference between experiment and theory is evident: the (110) face is not found experimentally, while the (010) faces was shown above to depend on the presence of additional molecules for stabilization.

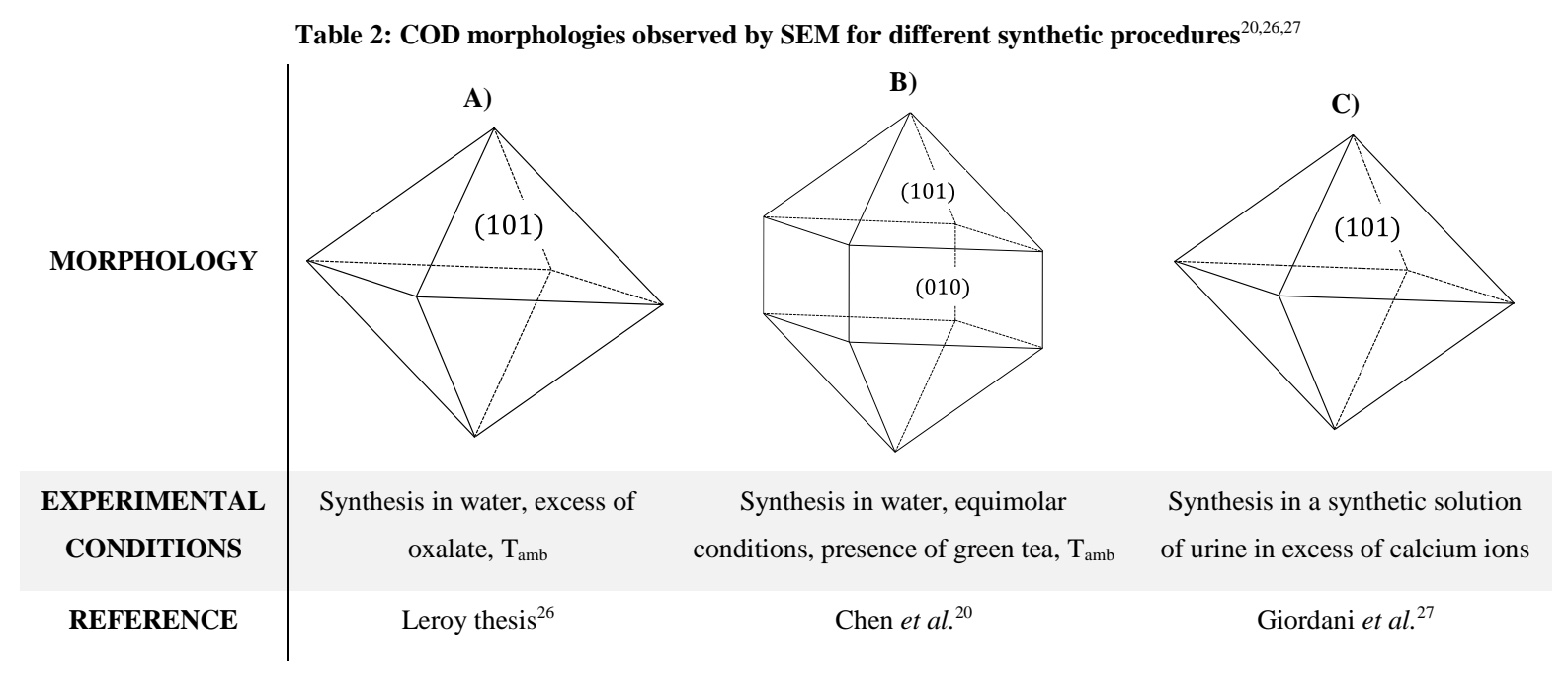

The scope of this contribution is to develop a theoretical approach able to predict the calcium oxalate growth in the presence of water with the aim of closing the gap between theoretical and experimental methods. The morphology prediction methods described above have strong limitations as they do not take into account possible rearrangements of ions present on the surface of the crystal. Furthermore, they do not explicitly take into account the effect of the solvent. These two contributions will affect the stabilization of particular faces and thus have an impact on the crystal morphology. To take into account these external factors explicitly, we have used total surface energy minimization in order to predict COM and COD morphologies, corresponding to the lowest total surface energy. Surface energies were calculated at a DFT 
level in the presence, or absence, of a solvation layer above the surfaces and with geometric relaxation. The development of such a model, will allow the subsequent study of the interaction between solvated COM/COD and additives at an atomic level with the aim of designing drugs that inhibit crystal growth.

\section{Methods}

Bulk structures of COM and COD were obtained from Daudon et al. ${ }^{12}$ (neutron diffraction) and Tazzoli et al. ${ }^{9}$ (X-ray diffraction), respectively. Calcium oxalate surfaces were built by adding a vacuum of $10 \AA$ in the bulk unit cell, along the low Miller indices (see Figure 1). 11 surface models, with different miller indices, were built for COM and 11 for COD by cutting along the bulk along different angles. The COM and COD surfaces are composed of 160 and 226 atoms, respectively.
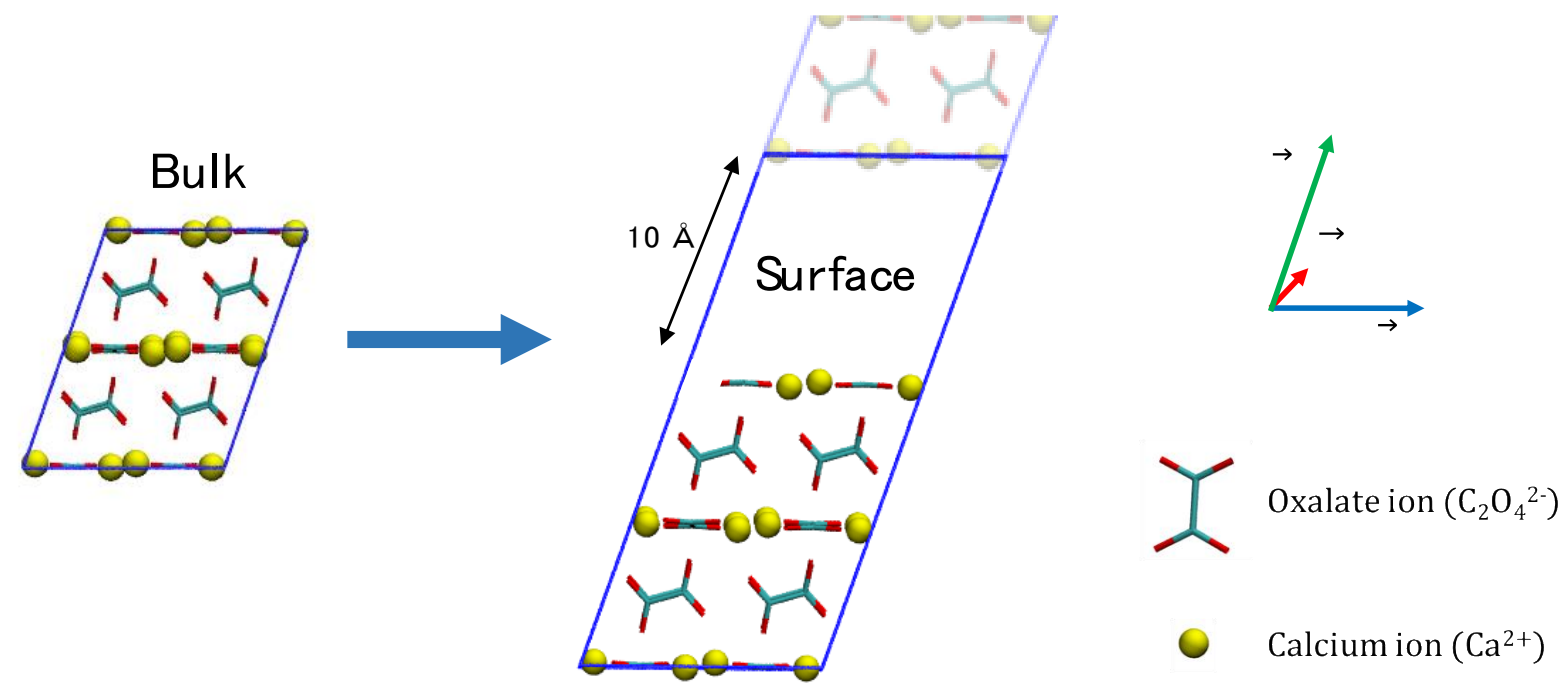

Figure 1: COM (100) surface construction from COM bulk. Initial data from Daudon et al. ${ }^{12}$

All geometry optimizations were carried out with the freely available DFT package CP2K/quickstep, ${ }^{32}$ based on the hybrid Gaussian and plane wave method ${ }^{33}$ using the generalized gradient approximation Perdew-Burke-Ernzerhof (PBE) + D3 functional. ${ }^{34}$ BLYP$\mathrm{D}^{35}$ and OptPBE-vdW ${ }^{36}$ functionals were tested in addition in order to compare surface energies calculations. The D3 Grimme method was used to account for the dispersion forces in the oxalate ions ${ }^{37}$. Analytic Goedecker-Teter-Hutter pseudopotentials ${ }^{38}$, a DZVP level basis set, and a density cutoff of 300 Ry were used. 
Water molecules were added to fill the vacuum present in each surface model super cell with the solvate command of the GROMACS simulation package (v 5.1.2) ${ }^{39}$ with an average density of $1 \mathrm{~kg} . \mathrm{L}^{-1}$. The Gromos force field $54 \mathrm{a} 7^{40}$ was used to describe interactions between oxalate and calcium ions and water molecules, while the SPC/E model ${ }^{41}$ was chosen for the latter. Calcium and oxalate positions were frozen using P-LINCS algorithm ${ }^{42}$ and a 500 ps simulation was performed to equilibrate the water molecules in the canonical ensemble (NVT) with a Nosé-Hoover thermostat to ensure thermal stability. ${ }^{43,44}$ The particle Mesh Ewald (PME) method was used to optimize calculation of long-range electrostatic interactions ${ }^{45,46}$ with a grid spacing of $0.12 \mathrm{~nm}$ and an interpolation of 4 . A real space cutoff of $0.5 \mathrm{~nm}$ was used, and the Lennard-Jones interactions were truncated at the same distance. For reasons of calculation costs, only the first two layers of solvation were kept for performing the geometry optimization of the hydrated surfaces at the DFT level. Indeed, one geometry optimization could take up to 48 hours of calculation on 128 cores, on the GENCI supercomputer, while a geometry optimization had to be performed for all "dry" and hydrated surface models, as well as for the bulk models. This led to 46 geometry optimizations in total.

The surface energy $\gamma_{\text {vacuum }}$ of dry surfaces was calculated as:

$$
\gamma_{\text {vacuum }}=\frac{E_{\text {surface }}-E_{\text {bulk }}}{2 A}
$$

with $A$ the surface area and $E_{\text {surface }}$ and $E_{\mathrm{bulk}}$ the potential energies of the surface and the bulk models, respectively. Hydrated surface energies were calculated as:

$$
\gamma_{\mathrm{H} 2 \mathrm{O}}=\frac{E_{\text {surface, } \mathrm{H} 2 \mathrm{O}}-E_{\mathrm{bulk}}-N \cdot E_{\mathrm{H}_{2} \mathrm{O}}}{2 A}
$$

whereby $N$ corresponds to the number of water molecules in the solvation layers. $E_{\text {surface,H2O }}$ is the potential energy of the solvated surface and $E_{\mathrm{H}_{2} \mathrm{O}}$ is the energy of one water molecule as obtained from an NVT Born Oppenheimer Molecular Dynamics simulation (BOMD) of 10 ps followed by a geometry optimization of 93 water molecules in a $10 \times 10 \times 10 \AA^{3}$ box with PBED3 functional. 
Crystal morphology predictions were made by means of Wulff diagrams and using either surface or hydrated surface energies. The predictions are based on the Gibbs hypothesis ${ }^{47}$ that the equilibrium shape of a crystal is the one minimizing the total surface energy of the crystal,

$$
\Delta G=\sum_{j} \gamma_{j} A_{j}
$$

with $\gamma_{j}$ the surface energy of a specific face, and $A_{j}$ the area of the face. $\Delta G$ then represents the difference in free energy between ions/molecules in a real crystal (with surfaces) and ions in an infinite crystal. Additionally, according to Gibbs ${ }^{48}$, the length of the vector $h_{j}$ normal to the face and drawn between the center of the crystal and the face, is proportional to the surface energy:

$$
h_{j}=k \cdot \gamma_{j}
$$

Equations 3 and 4 lead to the Wulff-Gibbs theorem which is applied here as implemented in the software developed by Zucker et al. ${ }^{49}$ specifically for that purpose.

Larger models were built from the DFT relaxed surface models in order to investigate the interface between predominant COM/COD surfaces and bulk water by using GROMOS force field $54 \mathrm{a} 7 .^{40}$ Indeed, the surface area was greatly increased by extension of the simulation box in two dimensions, parallel to the surface. These corresponding models have a surface 40 times larger than the DFT models and are composed of about 6000 atoms. Surface splitting was also increased from 10 to $50 \AA$. After addition of water molecules (to completely fill the vacuum), the simulation box contained around 50000 atoms. Water density profiles were calculated from $1 \mathrm{~ns}$ NVT simulations, while COM and COD atoms were constrained in the DFT equilibrium state.

\section{Results and discussion}

\section{The COM case}

Table 3 shows the resulting surface energies for surfaces in vacuum, compared to the corresponding energies upon addition of a bilayer of water. Using the surface energies in vacuum only the (010), (102) and (100) faces show up in the morphology prediction as shown 
in Figure 2, which contradicts experimental observations. Specifically, the emergence of the (100) face in the morphology prediction may be surprising as it is less stable than other surfaces not present in the prediction. This highlights the importance of the crystal shape in the Wulff prediction as an unexpected face may be preferred over others if the resulting total surface energy is smaller. Especially, the none-appearance of the (12 $\overline{1})$ face is striking as this is the second most stable surface in vacuum and was also reported in the oxalate crystal morphology literature. ${ }^{25}$ Another surprising observation may be the appearance of the (100) face - which is also observed experimentally - as its surface energy is high. However, the Wulff predictions and in fact natural processes alike - look for the most stable crystal shape and not for a simple combination of the most stable faces. This means that less stable faces may be preferred over others if this reduces the total surface energy of the crystal.

Table 3 : Surface Energies of Calcium Oxalate monohydrate (COM) in vacuum and with a bilayer of water.

\begin{tabular}{|c|c|c|}
\hline face & $\gamma_{\text {vacuum }}\left({\left.\mathrm{J} . \mathbf{M}^{-2}\right)}\right.$ & $\gamma_{\mathrm{H}_{2} \mathrm{O}}\left(\mathbf{J} \cdot \mathbf{M}^{-2}\right)$ \\
\hline$(\mathbf{1 2} \overline{\mathbf{1}})$ & 0.50 & 0.33 \\
\hline (021) & 0.62 & 0.39 \\
\hline (010) & 0.28 & 0.39 \\
\hline (001) & 0.63 & 0.42 \\
\hline (011) & 0.76 & 0.46 \\
\hline (102) & 0.52 & 0.51 \\
\hline (120) & 0.55 & 0.51 \\
\hline (012) & 0.65 & 0.59 \\
\hline (100) & 0.65 & 0.68 \\
\hline (101) & 1.19 & 0.91 \\
\hline (110) & 0.58 & 1.04 \\
\hline
\end{tabular}

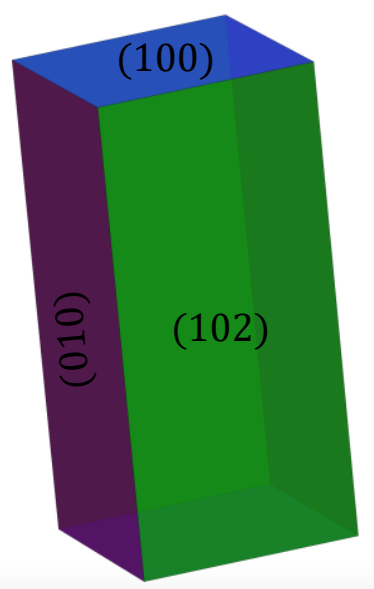

Figure 2: Morphology prediction of COM crystal in vacuum and based on $\gamma_{\text {vacuum }}$ values in Table 3. 
As suggested in the introduction, addition of a bilayer of water on the surfaces has a great impact on the surface energies. Indeed, the (12 $\overline{1})$ and (021) faces are strongly stabilized (by $-0.17 \mathrm{~J} . \mathrm{m}^{-}$ 2 and $-0.23 \mathrm{~J} . \mathrm{m}^{-2}$, respectively), whereas the (010) face is slightly destabilized $\left(+0.11 \mathrm{~J} . \mathrm{m}^{-2}\right)$. As shown in Figure 3 (in comparison with Figure 3), the solvation effect strongly affects the morphology, mainly due to the emergence of the $(12 \overline{1})$ face.
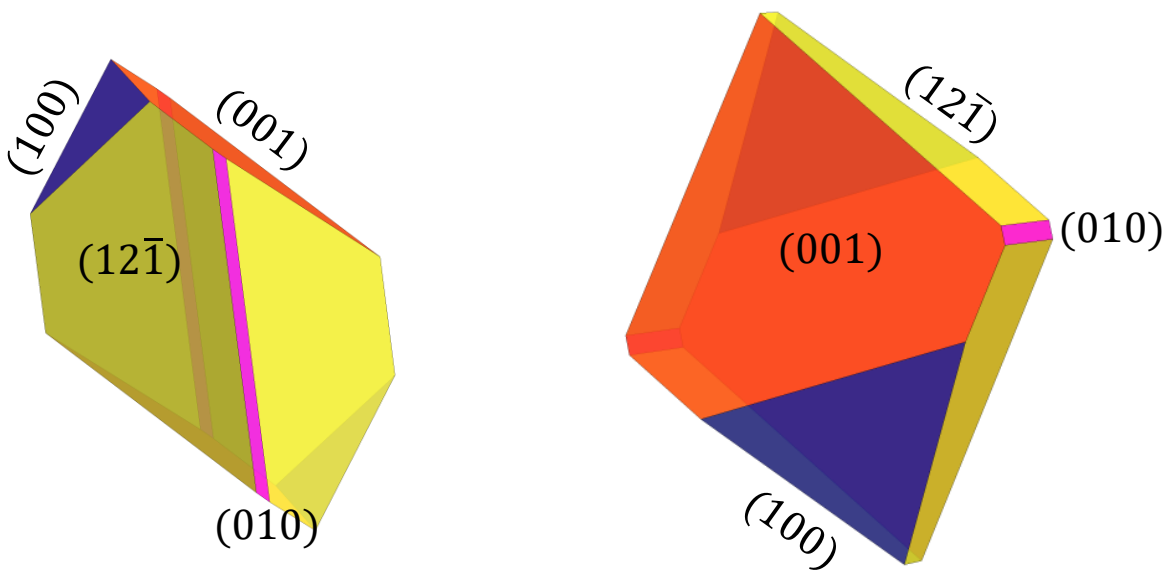

Figure 3: Morphology prediction of COM crystal with water molecules (bilayer), based on $\gamma_{\mathrm{H}_{2} \mathrm{O}}$ values in Table 3.

This morphology corresponds well to the Wulff prediction obtained with a force field by Tommaso et al. ${ }^{25}$ Nevertheless, a disagreement with experimental data remains as the (001) face is not observed in synthesized COM. As suggested by Milan ${ }^{16}$, the (001) face is not observed in experimental data due to kinetic effects and, as can be seen in Figure 4a, removing the (001) face from the Wulff prediction leads to a more satisfactory morphology. Still, when compared to experimental data (Table 1a and b), the presence of the (010) surface remains underestimated. As shown in Figure 4b, slightly decreasing the (010) surface energy (-0.1 J.m ${ }^{2}$ ) leads to a better prediction of the (010) surface exposure. Unfortunately, the software by Zucker et al. ${ }^{49}$ was not able to further increase the (010) face area in this way. It is important to note that the hydrated surface energies of the (12 $\overline{1}),(010)$ and (021) faces are very close to each other $\left(0.33,0.39\right.$ and $0.39 \mathrm{~J} . \mathrm{m}^{-2}$, respectively), falling within the error window of used methods. The expression of these faces in crystals are therefore expected to be very sensitive to external factors such as temperature, additives, solvent and so on. Indeed, as can be seen in Figure 5, a slight decrease of (010) and (021) surface energies (-0.03 J.m ${ }^{-2}$ ) causes the emergence of the (021) surface in the prediction leading to a close resemblance to the morphology found by Franchini-Angela et al. ${ }^{17,18}$ and with one of the mineral crystals in 
Erreur ! Source du renvoi introuvable.. As a summary, all relevant COM faces are shown in figure 7 .
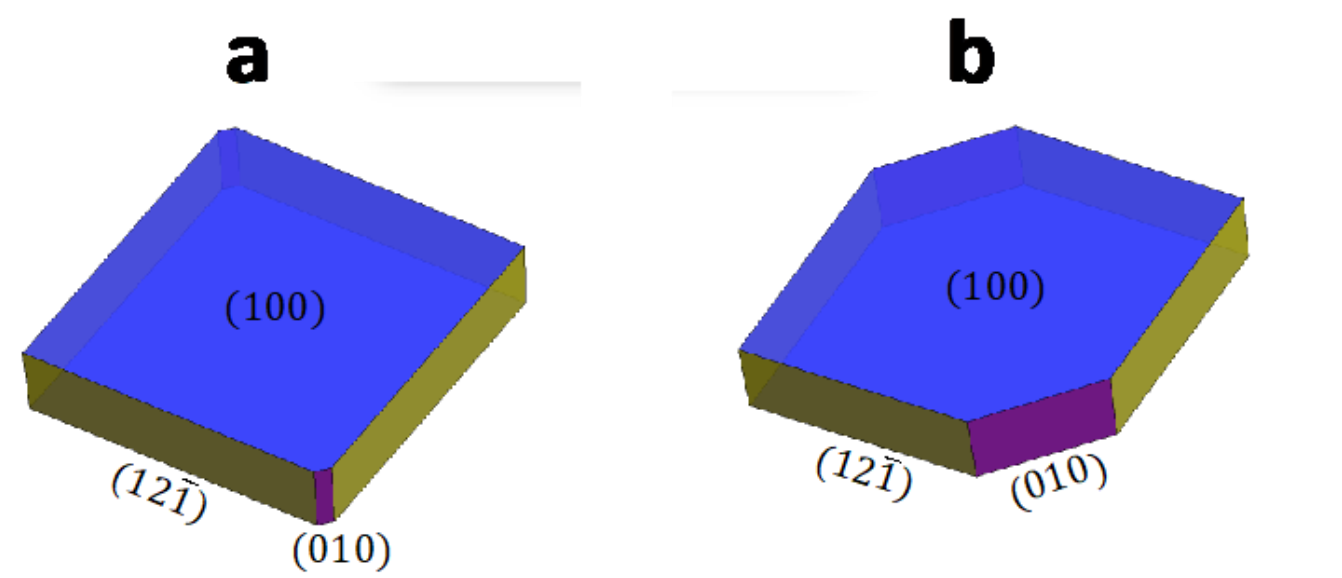

Figure $4:$ a) Morphology prediction of COM crystal with the water molecules, without (001) face. b) same but with a surface energy correction of $-0.1{\mathrm{~J} . \mathrm{m}^{-2}}^{-2}$ the (010) face.

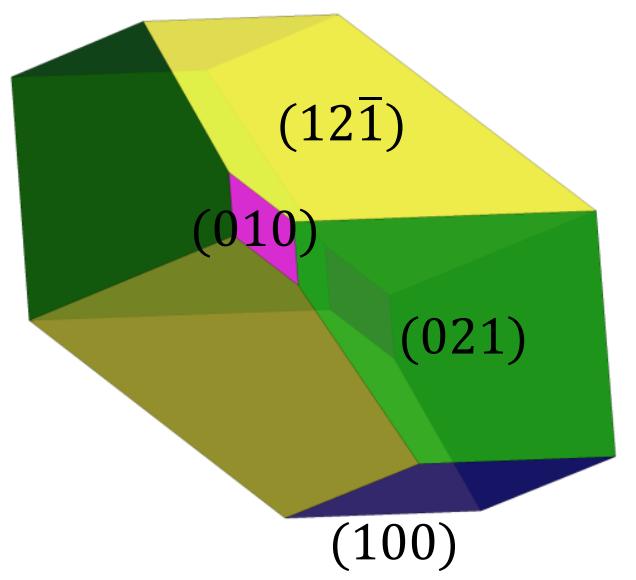

Figure 5 : Morphology prediction of COM crystal with water molecules, without (001) face and with energy corrections: -0.03 J.m ${ }^{-2}$ for (021) and (010) surface energies.

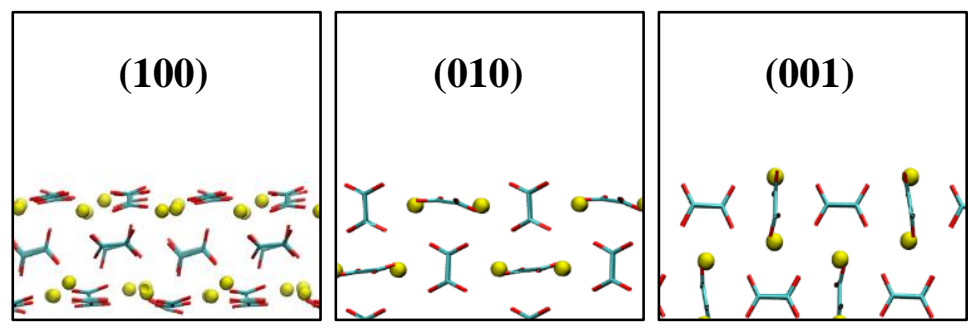




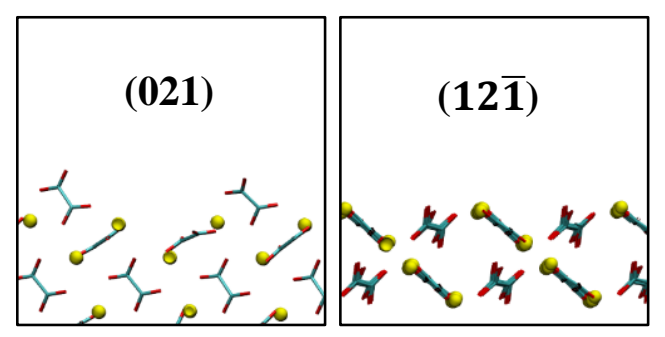

Figure 6: predominant COM faces

Next, the behavior of water over the COM surfaces was investigated by means of force field molecular dynamics simulations. Figure 7 represents the mean density profile of three entities: calcium ions, oxalate ions and water molecules, whereby the density of the water molecules coming from the COM structure (structural water) is left out. As said before, COM surface energies are strongly influenced by the presence of water which has an impact on the crystal shape: the (021), (12 $\overline{1})$ and (001) faces can be defined as hydrophilic $\left(\gamma_{\text {hydrated }}-\gamma_{\text {vacuum }}<\right.$ $0)$ while the (010) and (100) faces can be defined as hydrophobic $\left(\gamma_{\text {hydrated }}-\gamma_{\text {vacuum }}>0\right)$. This distinction can be perceived from the density profiles as in hydrophilic faces, water molecules are able to penetrate the surface in order to stabilize some under-coordinated ions leading to the appearance of water density peaks at in between the $\mathrm{Ca}^{2+}$ and oxalate ions density peaks. These particular $\mathrm{H}_{2} \mathrm{O}$ peaks are labeled with black arrows in Figure 7 and are only present in the hydrophilic faces. 

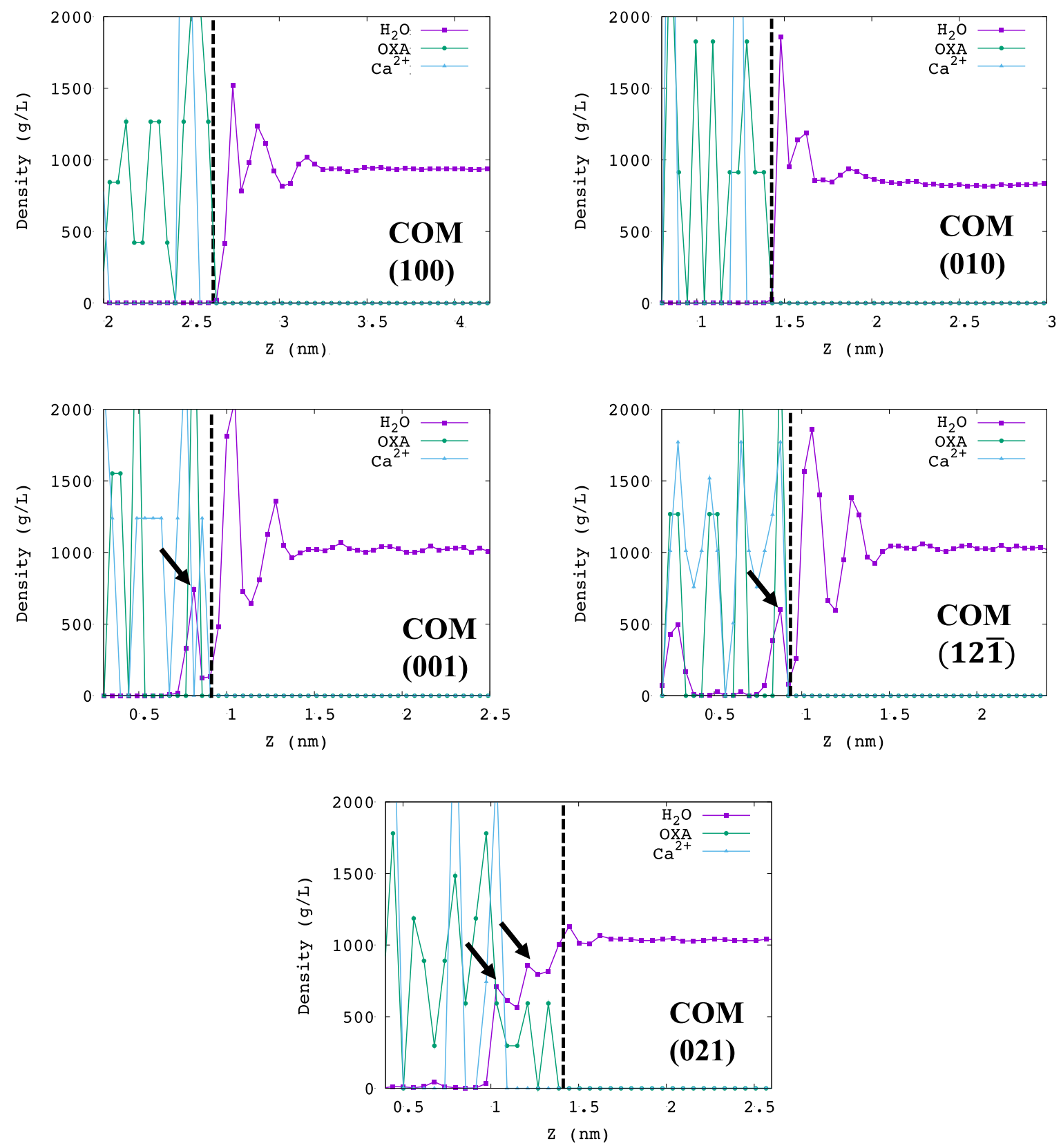

Figure 7: Density profile of water for COM (100), (010), (001) and (021) faces. Black arrows correspond to water molecules inside COM structure. $Z$ is the coordinate orthogonal to COM surface.

The corresponding solvation layers are shown in Figure 8. For the hydrophilic surfaces, (001), $(021)$ and $(12 \overline{1})$ faces, water molecules (labeled with black arrows) are cleary found to position themselves inside the COM structure. Because of the cutting of the bulk structure to obtain the respective surfaces, crystallographic sites where structural water was positioned in the bulk, are left empty at the surface. The water molecules from the solvated model can fill these holes and stablize the surface, lowering its surface energy. For the (100) and the (010) faces there are no holes in the structure, explaining why these faces are not stabilized by the use of a solvation 
model. By consequence, in order to correctly describe, at the very least, the hydrophilic COM surfaces and obtain their respective surface energies a solvation model is needed.

(100)

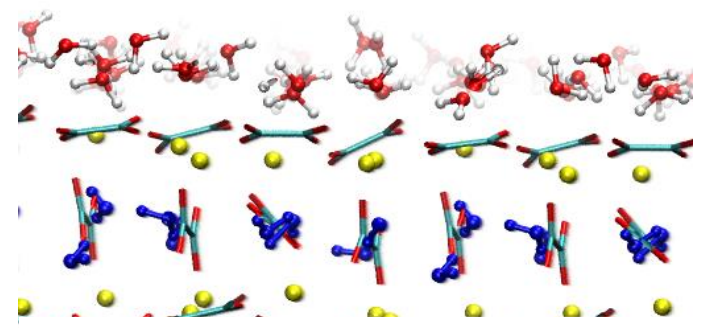

(001)

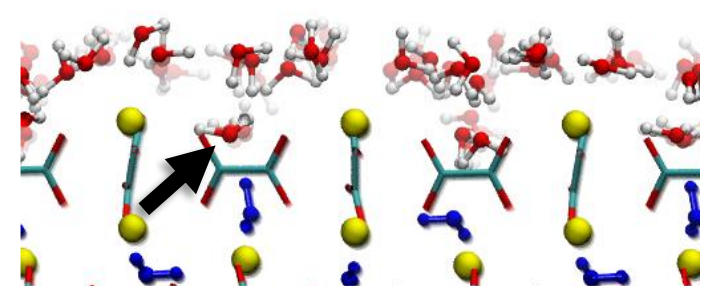

(010)

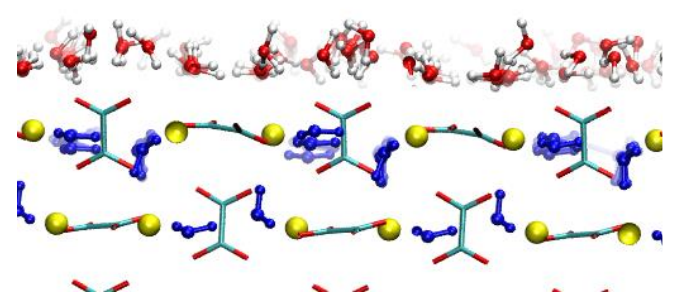

(021)

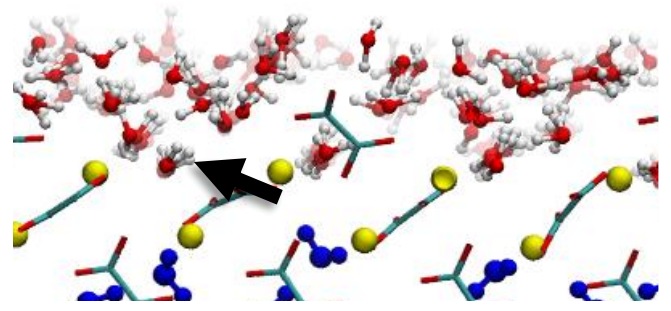

\section{$(12 \overline{1})$}

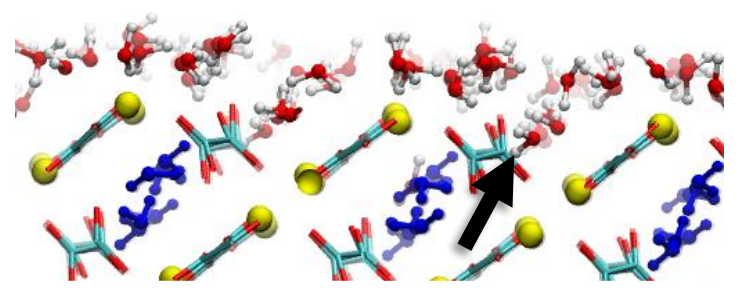

Figure 8: water molecules (red and white) interacting with COM (100), (010), (001), (021) and (12 $\overline{1})$ faces (blue : crystallographic water, yellow : $\mathrm{Ca}^{2+}$, green and red : oxalate). Blacks arrows corresponds to water molecules "inside" a given face.

\section{The COD case}

COD surface energies are reported in Table 4. The (101), (110), (010) and (011) faces are the most stable in vacuum as their surface energies are (almost) equal to each other and lower than all other calculated surfaces. These results lead to a rather correct morphology prediction (see Figure 9) in overall agreement with the theoretical morphology obtained with the attachment energies method. ${ }^{31}$ It is, however, in contrast with experimental observations where these four surfaces are energetically distinct. 


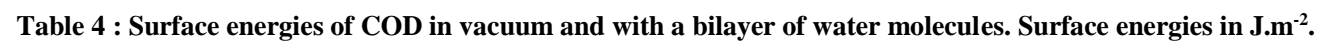

\begin{tabular}{|c|c|c|}
\hline face & $\gamma_{\text {vacuum }}$ & $\gamma_{\mathrm{H}_{2} \mathrm{O}}$ \\
\hline (101) & 0.29 & 0.24 \\
\hline (110) & 0.29 & 0.28 \\
\hline (010) & 0.29 & 0.35 \\
\hline (021) & 0.46 & 0.39 \\
\hline (001) & 0.47 & 0.41 \\
\hline (012) & 0.42 & 0.42 \\
\hline (111) & 0.59 & 0.42 \\
\hline (120) & 0.41 & 0.42 \\
\hline (112) & 0.46 & 0.44 \\
\hline (011) & 0.30 & 0.46 \\
\hline (121) & 0.48 & 0.46 \\
\hline
\end{tabular}

Adding a bilayer of water molecules changes the surface energies, leading to more realistic results. Indeed, solvation leads to a slight stabilization of the (101) face and a slight destabilization of the (010) face causing the disappearance of the (010) face in the morphology prediction (See Figure 10). This result is coherent with COD syntheses observations where the (010) face is observed only in the presence of specific additives (see Table 2b).

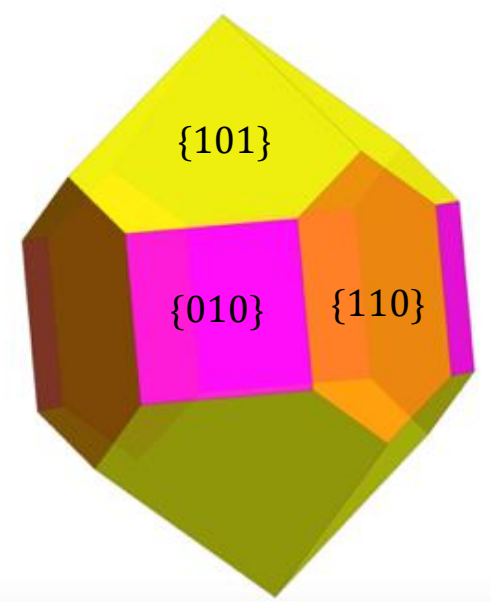

Figure 9: Morphology prediction of COD in vacuum and based on $\gamma_{\text {vacuum }}$ values in Table 4. 

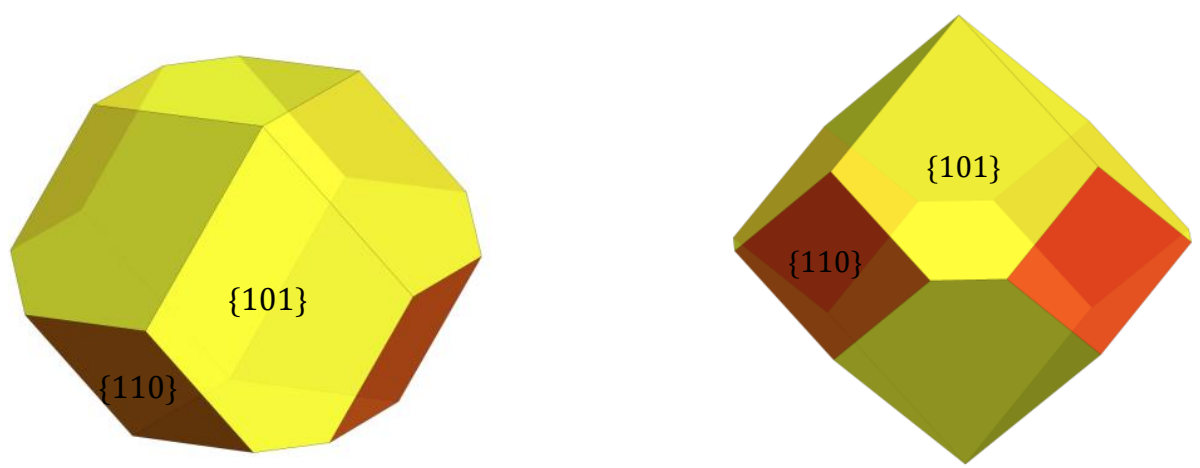

Figure 10 : Morphology prediction of hydrated COD and based on $\gamma_{\mathrm{H}_{2} \mathrm{O}}$ values in Table 4.

However, this prediction is still not in full agreement with experimental data (see Table 2a and Table 2c) where the (101) face is the only exposed face. Adding an energy correction of +0.2 $\mathrm{J} . \mathrm{m}^{-2}$ to the (110) face is necessary in order to obtain the morphology described in Figure 11, in close agreement with experimental observations. This energy is non-negligible suggesting that the COD crystal may not adopt the most stable morphology. Indeed, the (110) face is expected to be absent because of kinetics. The three relevant COD faces are presented in Figure 12.

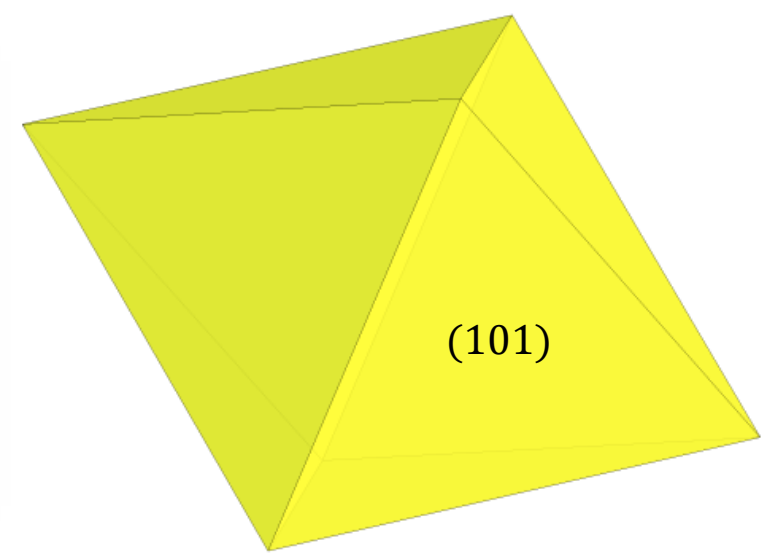

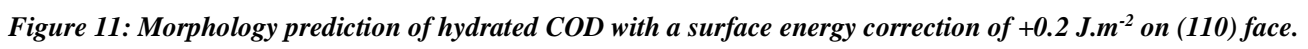
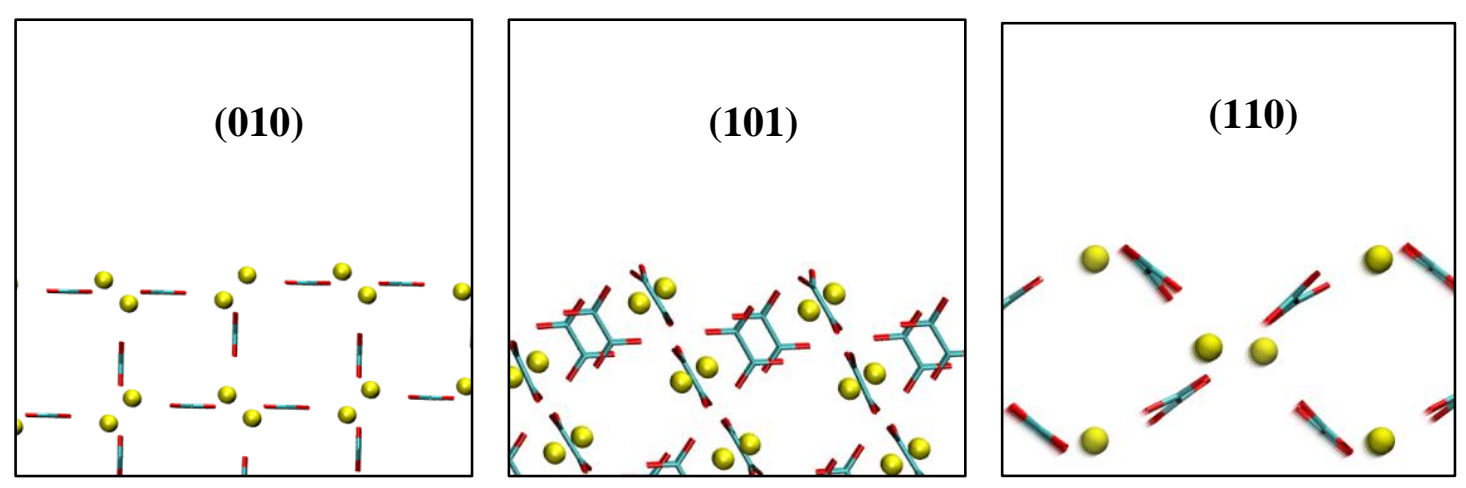
The molecular dynamics simulations show that, similar to the hydrophilic COM surfaces, water molecules penetrate inside the (101) face structure (black arrow in Figure 13 and Figure 14) to stabilize the surface. This solvation effect is not observed for the (010) face which may explain why the face is not observed in syntheses and needs specific conditions to be expressed (ie additional molecules). Finally, a density peak was also found inside the structure of the (110) face ( $c f$ Figure 13) but no particular stabilization was observed.
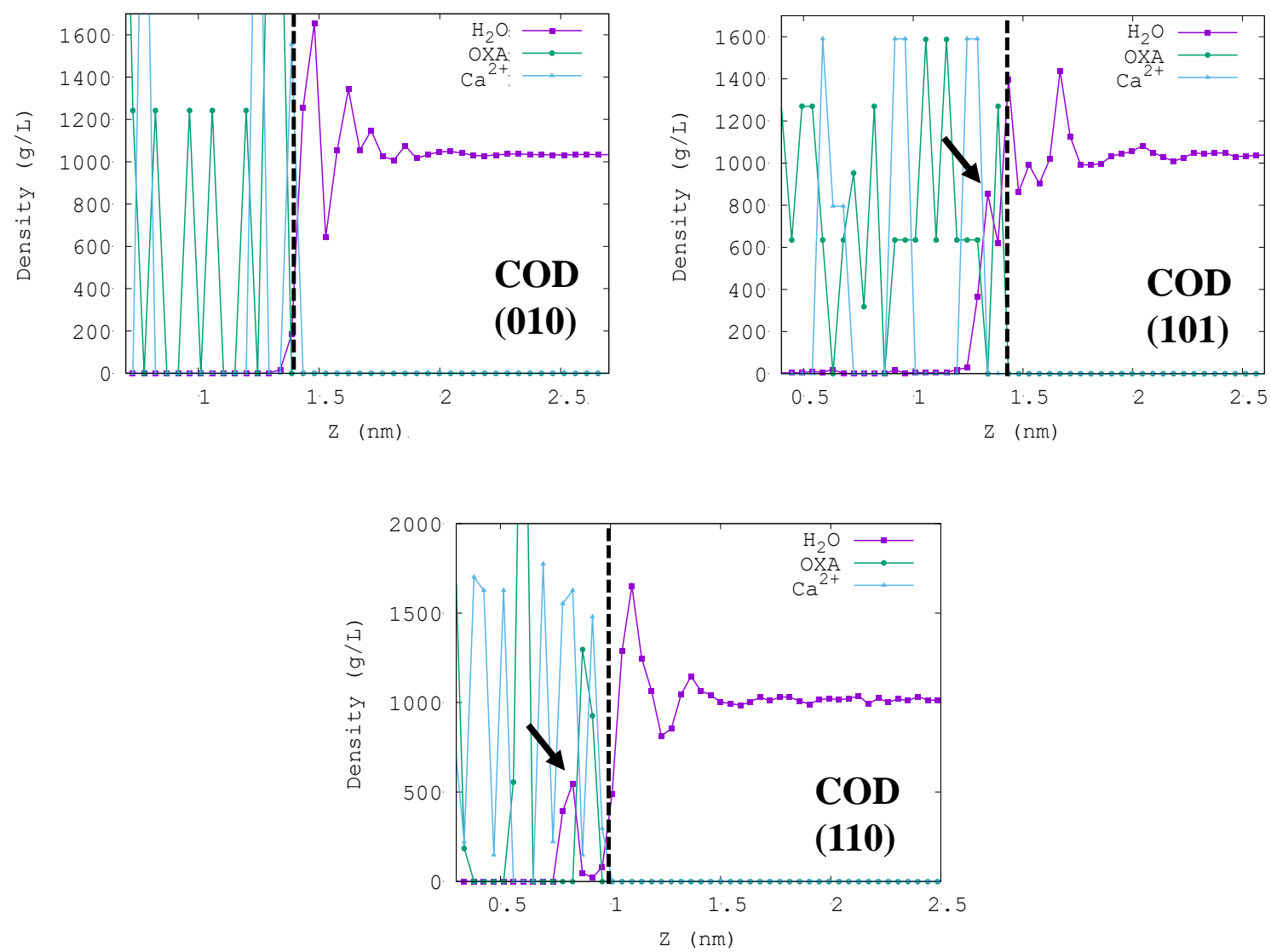

Figure 13: Density profile of water for COD 010,101 and 110 surfaces. Blacks arrows corresponds to water molecules inside COM structure. 


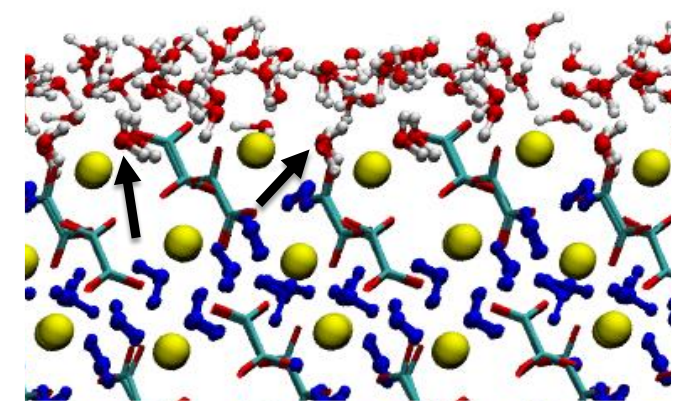

Figure 14: water molecules (red and white) filling empty water crystallographic sites at (101) interface (blue: crystallographic water, yellow: $\mathrm{Ca}^{2+}$, green and red: oxalate). Blacks arrows corresponds to water molecules inside COM structure.

\section{Conclusion}

The aim of this contribution is to develop a theoretical approach to predict the growth inhibition potential of selected molecules on calcium oxalate in a drug design perspective. Reliable models for COM and COD exposed faces, in agreement with experimental observations, were thus obtained by means of $a b$ initio total crystal surface energy minimization. It was found that a solvation model is crucial in order to obtain accurate surface energies and resulting morphologies. For most structures a very good agreement with experiment was obtained, while for some others the competition between thermodynamics and kinetics in the formation mechanism needs further investigation. In particular, the COM (001) face and the COD (110) face were predicted by our calculation while they are not observed experimentally. Removing those faces from the prediction was essential to obtain a morphology prediction in agreement with experiment. It is therefore clear that COM and COD crystals may not adopt the most thermodynamically stable morphology and are subject to kinetic effects. 


\section{Acknowledgements}

This work was performed using HPC resources from GENCI- [CCRT/CINES/IDRIS] (Grant 2016-[x2016082022]) and the CCRE of Université Pierre et Marie Curie. Computational resources and services were also provided by the Shared ICT Services Centre funded by the Vrije Universiteit Brussel, the Flemish Supercomputer Center (VSC) and FWO. FT wishes to acknowledge the VUB for support, among other through a Strategic Research Program awarded to his group.

\section{$\underline{\text { References }}$}

(1) Franceschi, V. R.; Nakata, P. A. Calcium Oxalates in Plants: Formation and Function. Annu. Rev. Plant Biol. 2005, $56 \quad$ (1), 41-71. https://doi.org/10.1146/annurev.arplant.56.032604.144106.

(2) Franceschi, V. R.; Horner, H. T. Calcium Oxalate Crystals in Plants. Bot. Rev. 1980, 46 (4), 361-427. https://doi.org/10.1007/BF02860532.

(3) Moe, O. W. Kidney Stones: Pathophysiology and Medical Management. Lancet 2006, 367 (9507), 333-344. https://doi.org/10.1016/S0140-6736(06)68071-9.

(4) Liu, Y.; Qu, M.; Carter, R. E.; Leng, S.; Ramirez-Giraldo, J. C.; Jaramillo, G.; Krambeck, A. E.; Lieske, J. C.; Vrtiska, T. J.; McCollough, C. H. Differentiating Calcium Oxalate and Hydroxyapatite Stones in Vivo Using Dual-Energy CT and Urine Supersaturation and PH Values. Acad. Radiol. 2013, 20 (12), 1521-1525. https://doi.org/10.1016/j.acra.2013.08.018.

(5) Hajir, M.; Graf, R.; Tremel, W. Stable Amorphous Calcium Oxalate: Synthesis and Potential Intermediate in Biomineralization. Chem. Commun. 2014, 50 (49), 6534-6536. https://doi.org/10.1039/C4CC02146K.

(6) Walton, R. C.; Kavanagh, J. P.; Heywood, B. R.; Rao, P. N. Calcium Oxalates Grown in Human Urine under Different Batch Conditions. J. Cryst. Growth 2005, 284 (3-4), 517529. https://doi.org/10.1016/j.jcrysgro.2005.06.057.

(7) Goldschmidt, V. Atlas der Krystallformen (1913 edition) | Open Library.

(8) Arnott, H. J.; Pautard, F. G. E.; Steinfink, H. Structure of Calcium Oxalate Monohydrate. Nature 1965, 208 (5016), 1197-1198. https://doi.org/10.1038/2081197b0.

(9) The Crystal Structures of Whewellite and Weddellite: Re-Examination and Comparison. Am. Mineral. 1980, 65 (3-4), 327-334. 
(10) Lieske, J. C.; Toback, F. G.; Deganello, S. Sialic Acid-Containing Glycoproteins on Renal Cells Determine Nucleation of Calcium Oxalate Dihydrate Crystals. Kidney Int. 2001, 60 (5), 1784-1791. https://doi.org/10.1046/J.1523-1755.2001.00015.X.

(11) Deganello, S.; IUCr. The Structure of Whewellite, CaC2O4. H2O at 328 K. Acta Crystallogr. Sect. B Struct. Crystallogr. Cryst. Chem. 1981, 37 (4), 826-829. https://doi.org/10.1107/S056774088100441X.

(12) Daudon, M.; Bazin, D.; André, G.; Jungers, P.; Cousson, A.; Chevallier, P.; Véron, E.; Matzen, G.; IUCr. Examination of Whewellite Kidney Stones by Scanning Electron Microscopy and Powder Neutron Diffraction Techniques. J. Appl. Crystallogr. 2009, 42 (1), 109-115. https://doi.org/10.1107/S0021889808041277.

(13) Sterling, C. Crystal Structure Analysis of Weddellite, CaC2O4.(2+x)H2O. Acta Crystallogr. 1965, 18 (5), 917-921. https://doi.org/10.1107/s0365110x65002219.

(14) Izatulina, A.; Gurzhiy, V.; Frank-Kamenetskaya, O. Weddellite from Renal Stones: Structure Refinement and Dependence of Crystal Chemical Features on H2O Content. Am. Mineral. 2014, 99 (1), 2-7. https://doi.org/10.2138/am.2014.4536.

(15) Bouropoulos, N.; Weiner, S.; Addadi, L. Calcium Oxalate Crystals in Tomato and Tobacco Plants: Morphology and in Vitro Interactions of Crystal-Associated Macromolecules. Chem. Eur. J. 2001, 7 (9), 1881-1888. https://doi.org/10.1002/15213765(20010504)7:9<1881::AID-CHEM1881>3.0.CO;2-I.

(16) Millan, A. Crystal Growth Shape of Whewellite Polymorphs: Influence of Structure Distortions on Crystal Shape. Cryst. Growth Des. 2001, 1 (3), 245-254. https://doi.org/10.1021/cg0055530.

(17) Aquilano, D.; Franchini-Angela, M. Twin Laws of Whewellite, CaC2O4.H2O. A Structural and Growth Approach. Phys. Chem. Miner. 1981, 7 (3), 124-129. https://doi.org/10.1007/BF00308228.

(18) Franchini-Angela, M.; Aquilano, D. Theoretical Growth Morphology of Whewellite CaC2O4.H2O. Phys. Chem. Miner. 1984, 10 (3), 114-120. https://doi.org/10.1007/BF00309645.

(19) Sandersius, S.; Rez, A. P. Morphology of Crystals in Calcium Oxalate Monohydrate Kidney Stones. https://doi.org/10.1007/s00240-007-0115-3.

(20) Chen, Z.; Wang, C.; Zhou, H.; Sang, L.; Li, X. Modulation of Calcium Oxalatecrystallization by Commonly Consumed Green Tea. Cryst. Eng. Comm 2010, 12 (3), 845-852. https://doi.org/10.1039/B913589H. 
(21) Haussühl, S. P. Hartman (Ed.): Crystal Growth: An Introduction. North-Holland Series in Crystal Growth Vol. 1. North-Holland Publishing Company, Amsterdam-London 1973. 531 S., Preis: \$ 21.10. Berichte der Bunsengesellschaft für Phys. Chemie 1974, 78 (11), 1276-1276. https://doi.org/10.1002/BBPC.19740781138.

(22) Docherty, R.; Clydesdale, G.; Roberts, K. J.; Bennema, P. Application of BravaisFriedel-Donnay-Harker, Attachment Energy and Ising Models to Predicting and Understanding the Morphology of Molecular Crystals. J. Phys. D. Appl. Phys. 1991, 24 (2), 89-99. https://doi.org/10.1088/0022-3727/24/2/001.

(23) Rijpkema, J. J. M.; Knops, H. J. F.; Bennema, P.; van der Eerden, J. P. Determination of the Ising Critical Temperature of F Slices with an Application to Garnet. J. Cryst. Growth 1983, 61 (2), 295-306. https://doi.org/10.1016/0022-0248(83)90366-4.

(24) Hartman, P.; Bennema, P. The Attachment Energy as a Habit Controlling Factor: I. Theoretical Considerations. J. Cryst. Growth 1980, 49 (1), 145-156. https://doi.org/10.1016/0022-0248(80)90075-5.

(25) Tommaso, D. Di; Hernández, S. E. R.; Du, Z.; Leeuw, N. H. de. Density Functional Theory and Interatomic Potential Study of Structural, Mechanical and Surface Properties of Calcium Oxalate Materials. RSC Adv. 2012, 2 (11), 4664. https://doi.org/10.1039/c2ra00832g.

(26) Leroy, C. Oxalates de Calcium et Hydroxyapatite: Des Matériaux Synthétiques et Naturels Étudiés Par Techniques RMN et DNP, Université P. et M. Curie, 2016.

(27) Giordani, P.; Modenesi, P.; Tretiach, M. Determinant Factors for the Formation of the Calcium Oxalate Minerals, Weddellite and Whewellite, on the Surface of Foliose Lichens. Lichenologist 2003, 35 (3), 255-270. https://doi.org/10.1016/S00242829(03)00028-8.

(28) Zhang, D.; Qi, L.; Ma, J.; Cheng, H. Morphological Control of Calcium Oxalate Dihydrate by a Double-Hydrophilic Block Copolymer. 2002. https://doi.org/10.1021/cm010768y.

(29) Bazin, D.; Leroy, C.; Tielens, F.; Bonhomme, C.; Bonhomme-Coury, L.; Damay, F.; Le Denmat, D.; Sadoine, J.; Rode, J.; Frochot, V.; Letavernier, E.; Haymann, J. P.; Daudon, M. Hyperoxaluria Is Related to Whewellite and Hypercalciuria to Weddellite: What Happens When Crystalline Conversion Occurs? Comptes Rendus Chim. 2016, 19 (1112), 1492-1503. https://doi.org/10.1016/j.crci.2015.12.011.

(30) Wesson, J. A.; Ward, M. D. Pathological Biomineralization of Kidney Stones. Elements 
2007, 3, 415-421.

(31) Heijnen, W. M. M.; Van Duijneveldt, F. B. The Theoretical Growth Morphology of Calcium Oxalate Dihydrate. J. Cryst. Growth 1984, 67 (2), 324-336. https://doi.org/10.1016/0022-0248(84)90192-1.

(32) VandeVondele, J.; Krack, M.; Mohamed, F.; Parrinello, M.; Chassaing, T.; Hutter, J. Quickstep: Fast and Accurate Density Functional Calculations Using a Mixed Gaussian and Plane Waves Approach. Comput. Phys. Commun. 2005, 167 (2), 103-128. https://doi.org/10.1016/J.CPC.2004.12.014.

(33) Lippert, G.; Hutter, J.; Parrinello, M. The Gaussian and Augmented-Plane-Wave Density Functional Method for Ab Initio Molecular Dynamics Simulations. Theor. Chem. Accounts Theory, Comput. Model. (Theoretica Chim. Acta) 1999, 103 (2), 124-140. https://doi.org/10.1007/s002140050523.

(34) Perdew, J. P.; Burke, K.; Ernzerhof, M. Generalized Gradient Approximation Made Simple. Phys. Rev. Lett. 1996, $77 \quad$ (18), 3865-3868. https://doi.org/10.1103/PhysRevLett.77.3865.

(35) Becke, A. D. Density-Functional Exchange-Energy Approximation with Correct Asymptotic Behavior. Phys. Rev. A 1988, 38 (6), 3098-3100. https://doi.org/10.1103/PhysRevA.38.3098.

(36) Klime, J.; Bowler, D. R.; Michaelides, A. Van Der Waals Density Functionals Applied to Solids. Phys. Rev. B - Condens. Matter Mater. Phys. 2011, 83 (19), 1-13. https://doi.org/10.1103/PhysRevB.83.195131.

(37) Grimme, S.; Antony, J.; Ehrlich, S.; Krieg, H. A Consistent and Accurate Ab Initio Parametrization of Density Functional Dispersion Correction (DFT-D) for the 94 Elements H-Pu. J. Chem. Phys. 2010, 132 (15), 154104. https://doi.org/10.1063/1.3382344.

(38) Goedecker, S.; Teter, M.; Hutter, J. Separable Dual-Space Gaussian Pseudopotentials. Phys. Rev. B 1996, 54 (3), 1703-1710. https://doi.org/10.1103/PhysRevB.54.1703.

(39) Abraham, M. J.; Murtola, T.; Schulz, R.; Páll, S.; Smith, J. C.; Hess, B.; Lindah, E. Gromacs: High Performance Molecular Simulations through Multi-Level Parallelism from Laptops to Supercomputers. SoftwareX 2015, 1-2, 19-25. https://doi.org/10.1016/j.softx.2015.06.001.

(40) Reif, M. M.; Hünenberger, P. H.; Oostenbrink, C. New Interaction Parameters for Charged Amino Acid Side Chains in the GROMOS Force Field. J. Chem. Theory 
Comput. 2012, 8 (10), 3705-3723. https://doi.org/10.1021/ct300156h.

(41) Berendsen, H. J. C.; Grigera, J. R.; Straatsma, T. P. The Missing Term in Effective Pair Potentials1. J. Phys. Chem 1987, 91, 6269-6271. https://doi.org/10.1021/j100308a038.

(42) Hess, B. P-LINCS: A Parallel Linear Constraint Solver for Molecular Simulation. J. Chem. Theory Comput. 2008, 3, 116-122. https://doi.org/10.1021/CT700200B.

(43) Nosé, S. A Molecular Dynamics Method for Simulations in the Canonical Ensemble. Mol. Phys. 1984, 52 (2), 255-268. https://doi.org/10.1080/00268978400101201.

(44) Hoover, W. G. Canonical Dynamics: Equilibrium Phase-Space Distributions. Phys. Rev. A 1985, 31 (3), 1695-1697. https://doi.org/10.1103/PhysRevA.31.1695.

(45) Darden, T.; York, D.; Pedersen, L. Particle Mesh Ewald: An $N \cdot \log (N)$ Method for Ewald Sums in Large Systems. J. Chem. Phys. 1993, 98 (12), 10089-10092. https://doi.org/10.1063/1.464397.

(46) Essmann, U.; Perera, L.; Berkowitz, M. L.; Darden, T.; Lee, H.; Pedersen, L. G. A Smooth Particle Mesh Ewald Method. J. Chem. Phys. 1995, 103 (19), 8577. https://doi.org/10.1063/1.470117.

(47) Gibbs, J. W. A. du texte. The Collected Works / of J. W. Gibbs,... 1928.

(48) Wulff, G. Zur Frage Der Geschwindigkeit Des Wachstums Und Der Auflösung Der Krystallflagen. Zeitschrift für Kryst. und Mineral. 1901, 34 (5/6), 449-530.

(49) Zucker, R. V.; Chatain, D.; Dahmen, U.; Hagège, S.; Carter, W. C. New Software Tools for the Calculation and Display of Isolated and Attached Interfacial-Energy Minimizing Particle Shapes. J. Mater. Sci. 2012, 47 (24), 8290-8302. https://doi.org/10.1007/s10853-012-6739-x. 


\section{Synopsis}

Reliable models for COM and COD exposed faces, in agreement with experimental observations, were obtained by means of ab initio total crystal surface energy minimization. It was found that a solvation model is crucial in order to obtain accurate surface energies and resulting morphologies. 